\title{
Overexpression of angiotensin II type 2 receptor suppresses neointimal hyperplasia in a rat carotid arterial balloon injury model
}

\author{
BING TANG* ${ }^{*}$, SHUANGTAO MA*, YONGJIAN YANG, DACHUN YANG, \\ JINSONG CHEN, XIAOHUA SU, YAN TAN, MEIQIN SUN and DE LI \\ Department of Cardiology, General Hospital of PLA Chengdu Military Area Command, Chengdu 610083, P.R. China
}

Received October 6, 2010; Accepted January 14, 2011

DOI: $10.3892 / \mathrm{mmr} .2011 .433$

\begin{abstract}
Angiotensin II (ANG II) type 2 receptor (AT2R) has been recognized to suppress the proliferation of vascular smooth muscle cells (VSMCs). The aim of the present study was to determine whether AT2R overexpression inhibits neointimal hyperplasia in a rat carotid arterial balloon injury model and to examine the underlying mechanisms of its activity. Balloon-injured rats receiving Ad-AT2R showed significant diminutions in neointimal area and intima/ media ratio compared to non-treated rats or rats receiving adenovirus containing green fluorescent protein (Ad-GFP). In addition, extracellular regulated kinase $1 / 2$ (ERK1/2) and basic transcription element-binding protein 2 (BTEB2) were significantly down-regulated in the arteries and VSMCs of Ad-AT2R-treated rats and compared to Ad-GFP-treated rats. However, Ad-AT2R transfection failed to affect the expression of ANG II type 1 receptor (AT1R) in carotid arteries and cultured VSMCs. The present study provides direct evidence that AT2R plays a beneficial role in balloon injury-induced neointimal hyperplasia, which is mainly attributed to the inhibition of VSMC proliferation and involves the down-regulation of the ERK1/2 and BTEB2 pathways, but is independent of the expression of AT1R.
\end{abstract}

\section{Introduction}

Percutaneous transluminal balloon angioplasty is widely used for the treatment of obstructed atherosclerotic vascular diseases (1). However, balloon injury to the arterial wall

Correspondence to: Dr De Li, Department of Cardiology, General Hospital of PLA Chengdu Military Area Command, Tianhui, Jinniu, Chengdu, Sichuan 610083, P.R. China

E-mail:dr_deli@yahoo.cn

\section{*Contributed equally}

Key words: angiotensin II type 2 receptor, neointimal hyperplasia, carotid arterial balloon injury induced by restenosis limits its overall benefits (2). Despite dramatic advances in reducing the incidence of restenosis by drug-eluting stents, it remains a considerable medical challenge (3-5). The major pathological process underlying restenosis is neointimal hyperplasia (6), which results from the phenotypic modulation, proliferation, migration and extracellular matrix synthesis of vascular smooth muscle cells (VSMCs) (7).

Release of angiotensin II (ANG II) in response to vascular injury plays an important role in neointimal hyperplasia through the stimulation of VSMC proliferation and migration $(8,9)$. Two distinct subtypes of ANG II receptors, type 1 (AT1R) and type 2 (AT2R), have been identified. The proliferation effect of ANG II has been attributed to AT1R (10,11). Additionally, treatment with AT1R blockers (ARBs) reduces the incidence of restenosis $(12,13)$. AT2R is expressed abundantly in the fetal vasculature with rapid decline after birth, and is re-expressed in the adult vasculature under certain pathological cardiovascular conditions, such as vessel injury and inflammation $(14,15)$. In injured vessels, AT1R is highly expressed, while AT2R is expressed at low levels (16). Furthermore, it has been reported that AT2R has anti-proliferation, anti-migration and proapoptotic effects on VSMCs in vitro, and antagonizes the growth effect of AT1R $(17,18)$. These lines of evidence suggest that AT2R plays a crucial role in the neointimal hyperplasia response to vascular injury.

In this study, we investigated the effects of the overexpression of AT2R by adenoviral gene transfer on neointimal hyperplasia in a rat carotid arterial balloon injury model, and explored the underlying mechanisms of its activity.

\section{Materials and methods}

Preparation of AT2R adenovirus vector. PUHD-AT2R (supplied by Dr Tao Jin, Department of Cardiology, Southwest Hospital, Chongqing, China) was transmitted to pAdTrackCMV, then the pAdEasy-1/AT2R was constructed. An adenovirus coding for AT2R and green fluorescent protein (GFP) (Ad-AT2R) was constructed as described previously (19). Ad-AT2R contains two expression cassettes for AT2R and GFP, both controlled by cytomegalovirus (CMV) promoters. The adenovirus containing GFP (Ad-GFP) was a 
gift from Dr Bert Vogelstein (John Hopkins Sarcoma Center, USA). The propagation and purification of both recombinant adenoviruses was performed in 293 cells (Chinese Academy of Sciences, Shanghai, China).

Animal model and gene transfer. The animal care and experimental protocols were approved by the Animal Care Committee of the General Hospital of PLA Chengdu Military Area Command. Adult male Sprague-Dawley rats (obtained from a local animal center) weighing 350-400 $\mathrm{g}$ were anesthetized with pentobarbital and heparinized with $100 \mathrm{U} / \mathrm{kg}$ heparin sodium. Balloon denudation was performed six times in the left common carotid artery with a 2-French catheter (Cordis, Miami, FL, USA) as previously described (20). Rats were randomly divided into four groups ( $\mathrm{n}=6$ per group). Rats in the control group were not injured with the balloon, while rats in the injury, Ad-GFP and Ad-AT2R groups received no treatment, a suspension of Ad-GFP $\left(1 \times 10^{8} \mathrm{pfu}\right)$ and a suspension of Ad-AT2R ( $\left.1 \times 10^{8} \mathrm{pfu}\right)$, respectively. After injury, the suspension was loaded in a $24-G$ indwelling needle and injected into the injured arterial segment, which was isolated with arterial clamps. After incubation for $30 \mathrm{~min}$, the needle and arterial clamps were removed. Rats were euthanized 21 days after injury. The blood vessels were harvested, rinsed with PBS and embedded for H\&E staining and immunohistochemical analysis.

VSMC culture and gene transfer. VSMCs were obtained from rat thoracoabdominal aortas and grown in high-glucose Dulbecco's modified Eagle's medium (DMEM; Hyclone, Logan, UT, USA) containing $10 \%$ fetal bovine serum (FBS; Hyclone) under standard culture conditions as previously described (21). Briefly, the aorta was thoroughly dissected to free it from the connective tissue and cut open longitudinally. The intimal and adventitial layers were scraped with a scalpel blade, and the aorta was cut into small pieces. Aortic explants were treated with collagenase and transferred into culture flasks. Cells were harvested for passaging at 2-week intervals, and cells between passages 3 and 5 were used for all experiments. The smooth muscle phenotype of the cultured cells was verified by positive immunofluorescence for smooth muscle $\alpha$-actin. VSMCs cultured in 6-well plates were divided into control, Ad-GFP and Ad-AT2R groups receiving no treatment, Ad-GFP $\left(1 \times 10^{6}\right.$ pfu per well $)$ and Ad-AT2R $\left(1 \times 10^{6} \mathrm{pfu}\right.$ per well), respectively. The cells were harvested 12, 24 and 48 $\mathrm{h}$ after transfection.

Histopathological analysis. Carotid arteries were harvested, embedded, sectioned, mounted on glass slides and conventionally stained with $\mathrm{H} \& \mathrm{E}$, and were then analyzed using a computer-assisted morphometric analysis system (Image Pro Plus 4.5 software; Media Cybernetics, Carlsbad, CA, USA) (22). The intimal and the medial areas and intima/media ratio were calculated.

Immunofluorescence. For the analysis of the expression of AT2R, the frozen carotid arteries were cut into cross-sections $(10 \mu \mathrm{m})$ and transferred onto glass slides, while the VSMCs were grown in chambers on glass slides. Arterial sections and cells were rinsed with PBS, fixed with ice-cold acetone for 15 min, incubated in blocking solution (10\% FBS) for $20 \mathrm{~min}$ at room temperature and incubated with rabbit antiAT2R (1:200 dilution; Santa Cruz Biotechnology, CA, USA) overnight at $4^{\circ} \mathrm{C}$. The sections and cells were washed and incubated with antibody conjugated to a fluorescent probe (rhodamine-conjugated goat anti-rabbit $\operatorname{IgG}$ (1:100 dilution; Santa Cruz Biotechnology) for $30 \mathrm{~min}$ at room temperature. After removing the unbound secondary antibodies by washing the preparations with PBS, imaging was performed using a fluorescence microscope (Nikon TE2000, USA) (23).

Immunohistochemical analysis. Paraffin-embedded arteries were cut into cross-sections $(5 \mu \mathrm{m})$, dewaxed and rehydrated. Incubation with primary antibodies was performed in a humidified chamber overnight at $4^{\circ} \mathrm{C}$ with the following antibodies: rabbit anti-AT2R (1:200 dilution; Santa Cruz Biotechnology), rabbit anti-AT1R (1:200 dilution; Zhongshan Biotech Ltd., Beijing, China), rabbit anti-ERK1/2 (1:200 dilution; Zhongshan Biotech Ltd.) and rabbit anti-BTEB2 (1:200 dilution; Santa Cruz Biotechnology). Sections were washed and incubated with a goat biotinylated anti-rabbit secondary antibody (1:200 dilution; Boster Co., Ltd., Wuhan, China), followed by incubation with streptavidin-biotin-peroxidase complex reagents from a SABC kit (Boster Co., Ltd.). A diaminobenzidine (DAB) staining kit (Boster Co., Ltd.) was used to detect a positive reaction by producing a brown color. Quantization of AT2R, AT1R, ERK1/2 and BTEB2 expression involved the use of an automated image analysis system (Image Pro Plus 4.5 software; Media Cybernetics, Carlsbad, CA, USA), and the positively stained area was measured and expressed as the mean percentage of the neointimal area in at least ten high-power fields (magnification, $\mathrm{x} 400$ ).

Western blot analysis. Protein lysates were obtained by homogenizing the carotid arteries and cultured cells with lysis buffer containing $1 \%$ Triton X-100, $150 \mathrm{mM} \mathrm{NaCl}$, $1 \mathrm{mM}$ EDTA, $2.5 \mathrm{mM}$ sodium pyrophosphate, $1 \mathrm{mM}$ $\beta$-glycerophosphate, $1 \mathrm{mM} \mathrm{Na}_{3} \mathrm{VO}_{4}, 1 \mu \mathrm{g} / \mathrm{ml}$ leupeptin, $1 \mu \mathrm{g} / \mathrm{ml}$ aprotinin and $20 \mathrm{mM}$ Tris (pH 7.5) (23). Protein concentration was determined with Bio-Rad protein assay reagent (Bio-Rad Laboratories, CA, USA). Equal amounts of protein from the extracts were separated by SDS-PAGE (12\%). The samples were then electroblotted onto a nitrocellulose membrane (Boehringer Mannheim Corporation, USA) and probed with antibodies against AT1R (1:500 dilution; Zhongshan Biotech Ltd.), ERK1/2 (1:500 dilution; Zhongshan Biotech Ltd.) and BTEB2 (1:500 dilution; Santa Cruz Biotechnology). After washing, the membrane was incubated with a horseradish peroxidase-conjugated secondary antibody (1:1,000 dilution; Santa Cruz Biotechnology) and bound antibody was visualized using a colored reaction. The relative band densities were quantified by densitometry using the Multi-Analyst software package (Bio-Rad Laboratories). Equal loading of protein was confirmed by measuring $\beta$-actin expression.

Semi-quantitative reverse-transcription-PCR. Total RNA was extracted from the carotid arteries and cultured VSMCs using TriPure reagent (Roche Diagnostics Corp., Indianapolis, IN, USA) (24). Total RNA ( $3 \mu \mathrm{g}$ ) was reverse-transcribed using a reverse transcription system (Promega, Madison, WI, USA). 
The cDNA was amplified by PCR using a Platinum Taq-DNA polymerase (Clontech, Palo Alto, CA, USA) following a standard semi-quantitative reverse-transcription-PCR technique in which the amplified products were not saturated at the number of cycles performed. The primers used for the amplification were as follows: AT1R sense 5'-CCT GAA GTA TCC GAT AGA AC-3', antisense 5'-GCC GAC TCC ATT CCA ATG AA-3' (product 277 bp); ERK1 sense 5'-AAC CTC CTG CTG AAC ACC AC-3', antisense 5'-CAG AGC CTG TTC AAC TTC AAT C-3' (product 464 bp); ERK2 sense 5'-AAC CTC CTG CTG AAC ACC AC-3', antisense 5'-CAG AGC CTG TTC AAC TTC AAT C-3' (product 270 bp); BTEB2 sense 5'-CGC GGA TCC ATG CCC AGT TCG ACA AA-3', antisense 5'-CCG GAA TTC CTC TGG TGG CGC TTC A-3' (product 662 bp); GAPDH-1 sense 5'-ACC ACA GTC CAT GCC ATC AC-3', antisense 5'-TCC ACC ACC CTG TTG CTG TA-3' (product 452 bp); GAPDH-2 sense 5'-GTG CTG AGT ATG TCG TGG AGT-3', antisense 5'-GGT GGT CCA GGG TTT CTT AC-3' (product 752 bp). The PCR products were visualized by electrophoresis in $1 \%$ agarose gel (Bio-Rad Laboratories) with ethidium bromide $(0.5 \mu \mathrm{g} / \mathrm{ml}$; Sigma, MO, USA).

Statistical analysis. Data are presented as the means \pm standard deviation. Comparisons between groups were determined by one-way ANOVA with Student's post hoc t-test (SPSS Inc., Chicago, IL, USA). Results were considered significant when the p-value was $<0.05$.

\section{Results}

Expression of AT2R in arteries and VSMC. The protein expression of AT2R was undetectableby immunohistochemistry in carotid arteries from non-injured rats (control group, Fig. 1A). Balloon injury slightly stimulated the expression of AT2R (Fig. 1A). Immunohistochemistry showed a higher level of AT2R protein expression in the Ad-AT2R group relative to the Ad-GFP group ( $\mathrm{p}<0.01$, Fig. 1A). Similar results were obtained using the immunofluorescence assay (Fig. 1B). The experiment conducted in vitro demonstrated that AT2R protein expression detected by immunofluorescence was increased in the cultured VSMCs of the Ad-AT2R group $24 \mathrm{~h}$ after transfection, as compared to the Ad-GFP group (Fig. 1C).

AT2R attenuates intimal hyperplasia. As expected, the rats with balloon injury presented significantly with neointimal hyperplasia in the carotid artery (Fig. 2A and B). Balloon-injured rats receiving Ad-AT2R showed a significant diminution in neointimal area compared to non-treated rats (control group) or rats receiving Ad-GFP $(\mathrm{p}<0.01)$, indicating the beneficial effect of AT2R on neointimal hyperplasia (Fig. 2A and B). Similarly, the ratio of intimal to medial area was significantly reduced in Ad-AT2R-treated arteries compared to non-treated or Ad-GFP-treated arteries $(\mathrm{p}<0.01$, Fig. 2B). There were no significant differences in the medial area among the four groups (Fig. 2B).

$A T 2 R$ regulates ERK1/2 and BTEB2 in vivo. AT1R, ERK1/2 and BTEB2 protein expression levels in carotid arteries were examined by immunohistochemistry. Ad-AT2R transfection

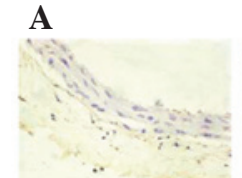

Control

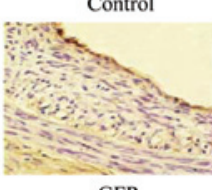

GFP

B
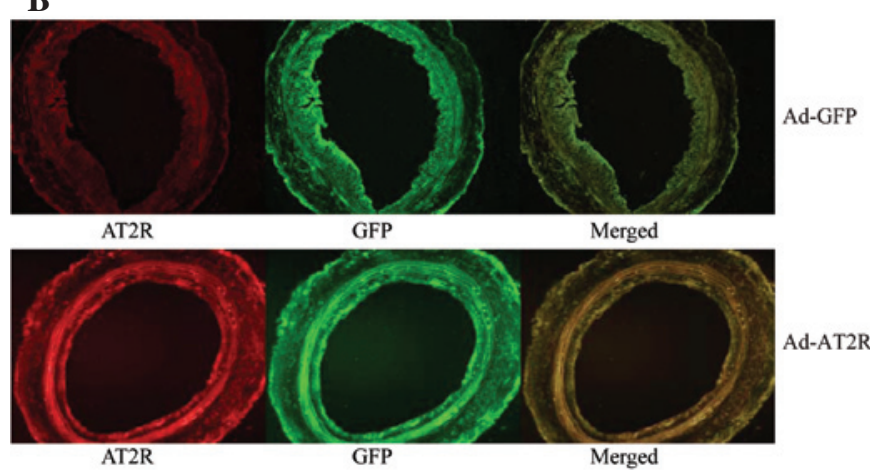

C
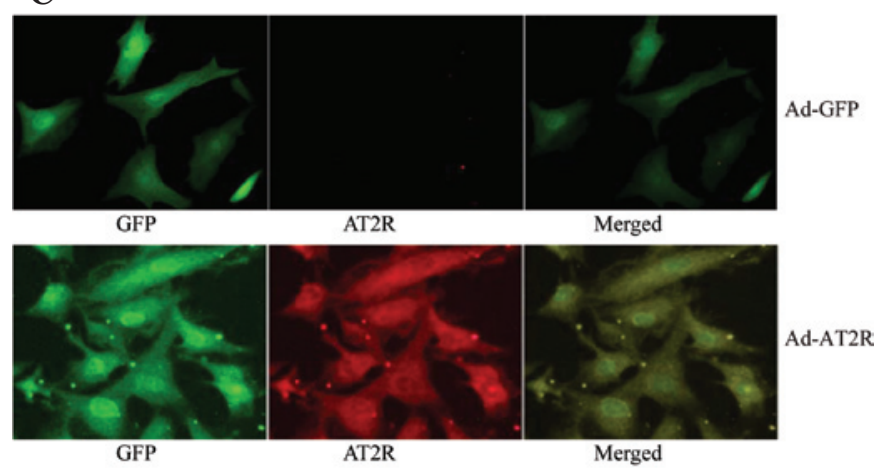

Figure 1. Expression of AT2R in carotid arteries and cultured VSMCs. (A) Protein expression of AT2R in carotid arteries from rats in control, nontreated (injury), Ad-GFP-treated and Ad-AT2R-treated groups was detected by immunohistochemistry. ${ }^{* *} \mathrm{p}<0.01, \mathrm{n}=6$. The overexpression of AT2R in (B) carotid arteries and (C) cultured VSMCs after Ad-AT2R transfection was confirmed by immunofluorescence. Green, GFP; red, AT2R; yellow, merged.

failed to affect the expression of AT1R in carotid arteries (Fig. 3A and E). ERK1 and ERK2 were significantly downregulated in the Ad-AT2R group compared to the Ad-GFP group (both $\mathrm{p}<0.01$, Fig. 3B, C, F and G). Similarly, the expression of BTEB2 was markedly suppressed after transfection with Ad-AT2R compared to the Ad-GFP-treated arteries $(\mathrm{p}<0.01$, Fig. 3D and H).

$A T 2 R$ regulates $E R K 1 / 2$ and $B T E B 2$ in vitro. Consistent with the in vivo experiment, the mRNA and protein expression of AT1R was not affected by the overexpression of AT2R (Fig. 4A and D). The mRNA and protein expression of ERK1 and ERK2 was markedly reduced in cultured VSMC after transfection with Ad-AT2R for 12-48 $\mathrm{h}$ compared to the Ad-GFP-treated VSMC ( $\mathrm{p}<0.05$ or $\mathrm{p}<0.01$, Fig. 4B, E and $\mathrm{F})$. In addition, the mRNA and protein expression of BTEB2 
A
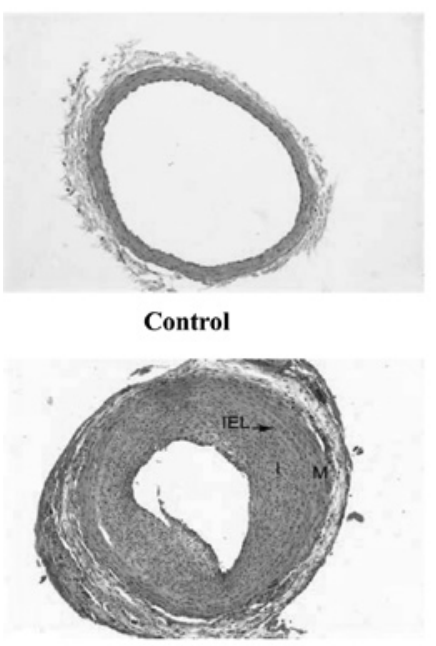

GFP
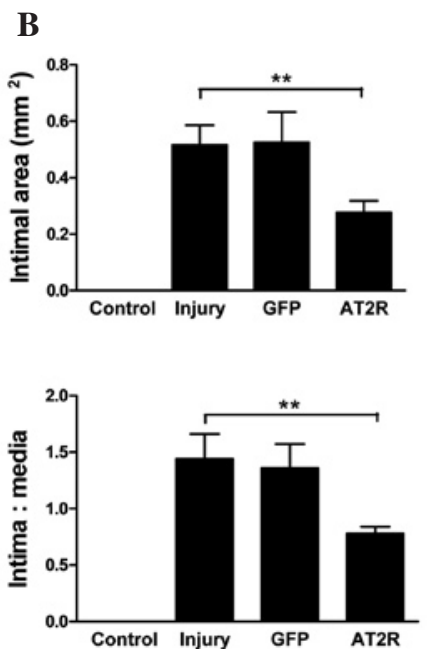

Figure 2. Effect of AT2R overexpression on neointimal hyperplasia in carotid arteries 21 days after injury. (A) H\&E-stained cross-sections of carotid arteries from rats in control, non-treated (injury), Ad-GFP-treated and Ad-AT2R-treated groups. I, intima; M, media; IML, internal elastic lamina. (B) Intimal and medial cross-sectional area and the ratio of the intimal area to the medial area of the injured arteries in the four groups $(n=6$ for each group) were calculated. Data are expressed as the mean $\pm \mathrm{SD},{ }^{* *} \mathrm{p}<0.01$.

was significantly suppressed in the Ad-AT2R-treated VSMC compared to the Ad-GFP-treated cells $(\mathrm{p}<0.05$ and $\mathrm{p}<0.01$, Fig. 4C and G).

\section{Discussion}

The vascular effect of ANG II is dependent on the opposing action of its two receptors, ATR1 and ATR2 (25). The latter is thought to counterbalance AT1R by providing some vascular protective effects (17). ANG II-induced cell proliferation is mostly mediated by AT1R (10). However, the potential role of AT2R in cell proliferation and neointimal hyperplasia and its precise mechanisms have not been fully characterized (26). In the present study, we focused on the function of AT2R in cell proliferation, as well as on its potential role in neointimal hyperplasia. The key finding of this study is that local overexpression of AT2R significantly inhibits the development of neointimal hyperplasia in a rat carotid arterial balloon injury
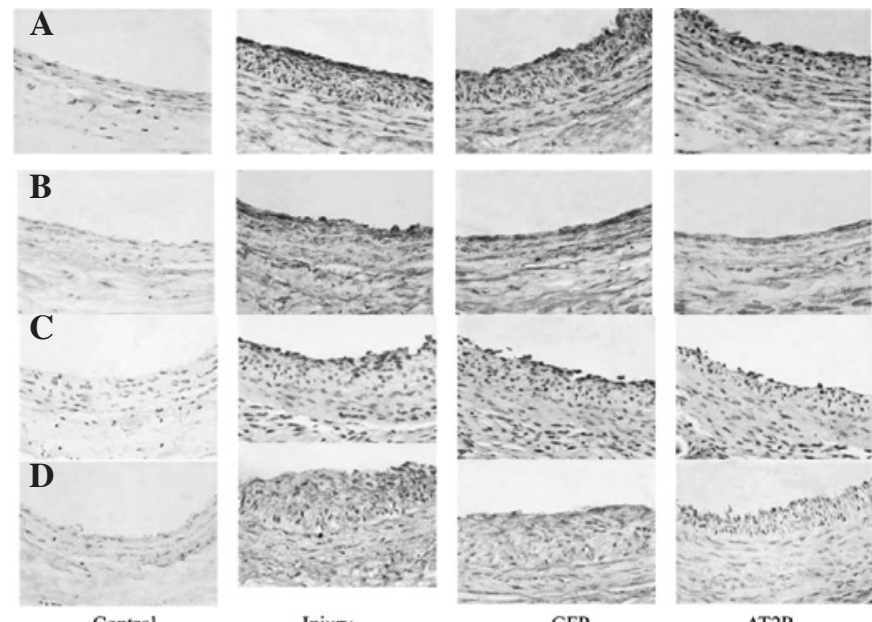

Injury

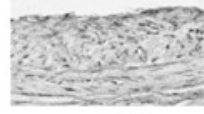

GFP

AT2R

$\mathbf{E}$

$\mathbf{F}$
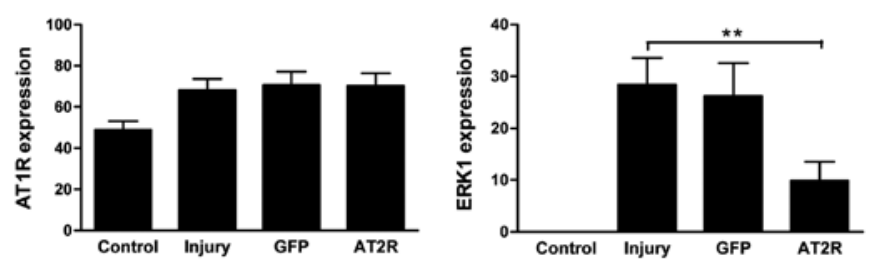

G

H
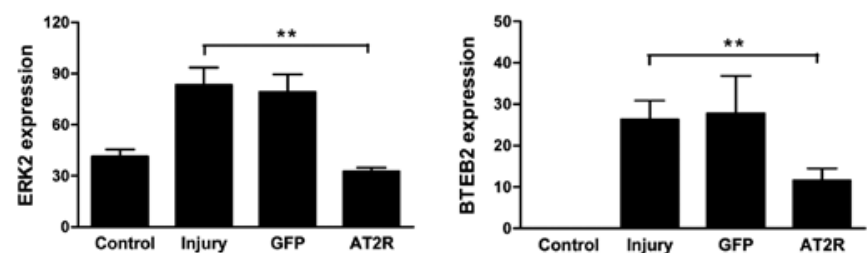

Figure 3. Effect of AT2R on ERK1/2 and BTEB2 expression in carotid arteries. Representative immunohistochemistry images of carotid arteries from rats in control, non-treated (injury), Ad-GFP and Ad-AT2R groups with antibodies to AR1R, ERK1/2 and BTEB2 (A-D). Quantification for the expression of AR1R, ERK1/2 and BTEB2 (E-H). Data are expressed as the mean $\pm \mathrm{SD},{ }^{* *} \mathrm{p}<0.01$.

model. The anti-neointimal hyperplasia effect of AT2R is mainly attributed to the inhibition of VSMC proliferation. The underlying mechanism involves the down-regulation of the ERK1/2 and BTEB2 pathways, but is independent of the expression of AT1R.

ANG II-induced neointimal growth and restenosis in injured arteries has been well documented (8). The infusion of ANG II in rats has been shown to accelerate intimal thickening in balloon-injured carotid arteries (17). The intimal thickening caused by injury in the rat carotid artery is inhibited by AT1R antagonists (12), suggesting an AT1R-mediated effect of ANG II in this process. The effect of AT2R, the other receptor of ANG II, on ANG II-induced neointimal hyperplasia has yet to be elucidated. A previous study showed that infusion of PD123319, a specific AT2R antagonist, does not affect ANG II-induced neointimal thickening (13), indicating that AT2R is not involved in this process. However, whether AT2R plays a protective role in neointimal thickening has not been conclusively determined. The present study provides direct evidence that AT2R plays a beneficial role in balloon injury-induced neointimal hyperplasia.

A previous study demonstrated that the anti-proliferative effect of AT2R may be mediated by antagonizing the growth 


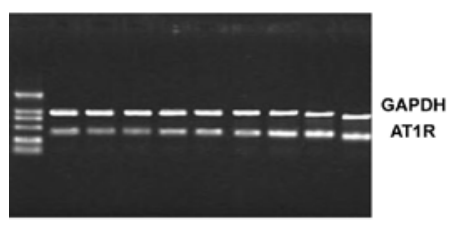

M C G A $\frac{C \text { G A }}{12 \mathrm{~h}} \frac{C \mathrm{~A}}{24 \mathrm{~h}}$

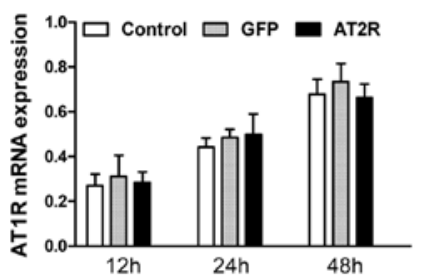

C

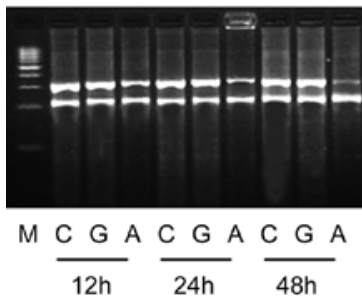

D
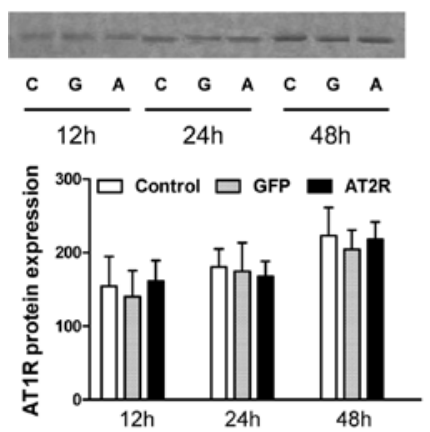

F
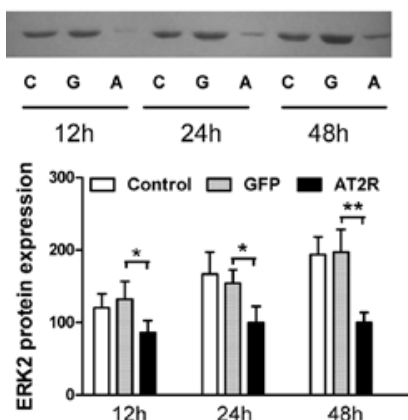

B

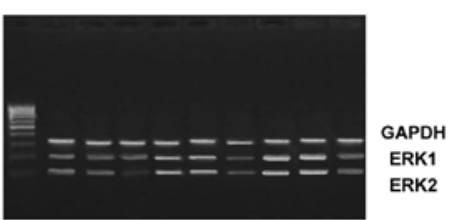

$M \frac{C G A}{12 h} \frac{C G A}{24 h} \frac{C G A}{48 h}$
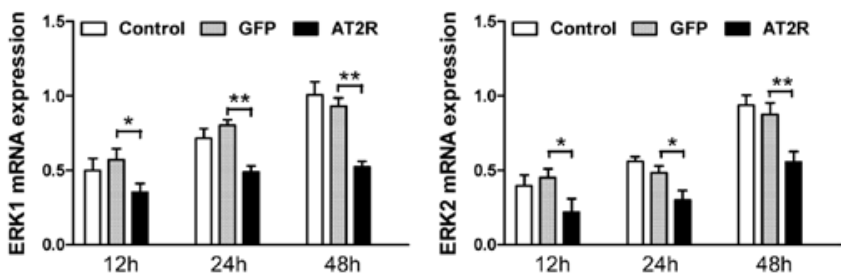

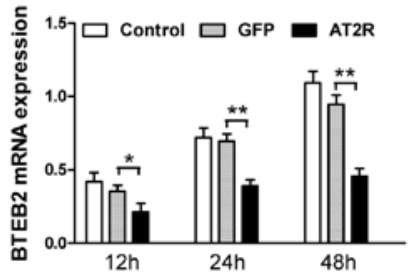

E
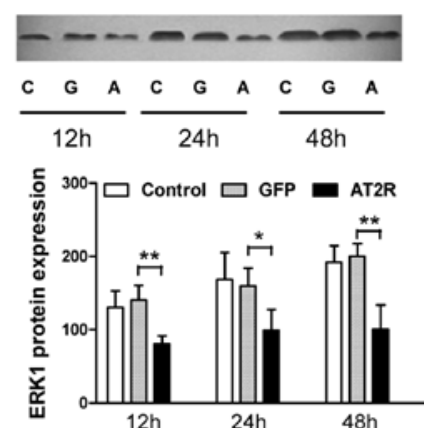

G
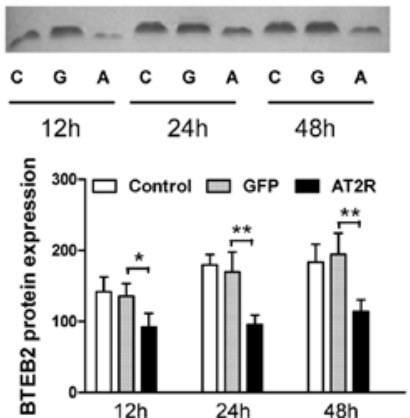

Figure 4. Effect of AT2R on ERK1/2 and BTEB2 expression in cultured VSMCs. The mRNA expression of AR1R, ERK1/2 and BTEB2 in cultured VSMCs from control, Ad-GFP and Ad-AT2R groups was detected by RT-PCR (A-C). Target molecules/GAPDH ratios are shown in the bar graphs. The protein expression of AR1R, ERK1/2 and BTEB2 in cultured VSMCs from control, Ad-GFP and Ad-AT2R groups was detected by Western blotting (D-F). Equal protein loading was confirmed using $\beta$-actin antibody. The band density is shown in the bar graph. M, marker; C, control; G, GFP; A, AT2R. Data are expressed as the mean $\pm \mathrm{SD},{ }^{*} \mathrm{p}<0.05,{ }^{* *} \mathrm{p}<0.01$.

effects of AT1R (17). However, the present study showed that the expression of AT1R was not affected by the overexpression of AT2R, indicating that AT1R may not be involved in the anti-proliferative effect of AT2R. It has been reported that the beneficial effects of valsartan on inflammation-induced vascular injury are less prominent in AT2R-deficient mice (14). These findings suggest that the beneficial effect of antiAT1R is dependent on the up-regulation of AT2R, whereas 
the beneficial effect of AT2R overexpression is not linked to the down-regulation of AT1R.

Several molecules, including ERK1 and ERK2, have been involved in ANG II-induced VSMC proliferation (27). AT2R is abundantly expressed in VSMC of the fetal vasculature during late gestation (15). The expression of AT2R during fetal vasculogenesis influences the growth phenotype of VSMC via the modulation of the ERK cascade (28). The present study demonstrated that AT2R-induced suppression of intimal hyperplasia is associated with the down-regulation of ERK1/2. It has also been reported that AT2R inhibits the activity of ERK. Several observations indicate that the ERK pathway activates the BTEB2 promoter (29). The present study found that the inhibition of ERK1/2 is linked to the downregulation of BTEB2. These findings suggest that ERK1/2 and BTEB2 are responsible for the therapeutic effects of AT2R on intimal hyperplasia.

In conclusion, we showed that AT2R is a critical regulator of VSMC proliferation and neointimal hyperplasia. AT2R may represent a novel therapeutic target in the prevention of restenosis after vascular intervention.

\section{Acknowledgements}

The authors thank Dr Tao Jin of Southwest Hospital for the supply of the plasmid PUHD-AT2R, and Dr Bert Vogelstein of John Hopkins Sarcoma Center for the gift of adenovirus vector Ad-GFP. This study was supported by the Foundation for the Talents by the General Hospital of PLA Chengdu Military Area Command.

\section{References}

1. Lin GA, Dudley RA and Redberg RF: Cardiologists' use of percutaneous coronary interventions for stable coronary artery disease. Arch Intern Med 167: 1604-1609, 2007.

2. Nobuyoshi M, Kimura T, Ohishi $\mathrm{H}$, et al: Restenosis after percutaneous transluminal coronary angioplasty: pathologic observations in 20 patients. J Am Coll Cardiol 17: 433-439, 1991.

3. Er F and Erdmann E: Drug-eluting or bare-metal stents in interventional cardiology. Theory and practice (in German). Med Klin 102: 30-32, 2007.

4. Corbett SJ, Cosgrave J, Melzi G, et al: Patterns of restenosis after drug-eluting stent implantation: insights from a contemporary and comparative analysis of sirolimus- and paclitaxel-eluting stents. Eur Heart J 27: 2330-2337, 2006.

5. Li D, Ma S, Li G, et al: Endovascular stent implantation for isolated pulmonary arterial stenosis caused by Takayasu's arteritis. Clin Res Cardiol 99: 573-575, 2010.

6. Shah PK: Inflammation, neointimal hyperplasia, and restenosis: as the leukocytes roll, the arteries thicken. Circulation 107: 2175-2177, 2003.

7. Xu L, Wu Y, Feng G, et al: Endovascular irradiation prevents smooth muscle cell proliferation and neointimal hyperplasia in rabbits. J Tongji Med Univ 19: 240-245, 1999.

8. Li F, Zhang C, Schaefer S, Estes A and Malik KU: ANG II-induced neointimal growth is mediated via cPLA2- and PLD2-activated Akt in balloon-injured rat carotid artery. Am J Physiol Heart Circ Physiol 289: H2592-H2601, 2005.

9. Hutchinson HG, Hein L, Fujinaga M and Pratt RE: Modulation of vascular development and injury by angiotensin II. Cardiovase Res 41: 689-700, 1999.
10. Van Kleef EM,Fingerle J and Daemen MJ: Angiotensin II-induced progression of neointimal thickening in the balloon-injured rat carotid artery is AT1 receptor mediated. Arterioscler Thromb Vasc Biol 16: 857-863, 1996.

11. Harada K, Komuro I, Sugaya T, Murakami K and Yazaki Y: Vascular injury causes neointimal formation in angiotensin II type 1a receptor knockout mice. Circ Res 84: 179-185, 1999.

12. Zhu J and Gao D: Losartan reduces collagen content and intimal thickening of iliac arteries after balloon injury in rabbits. J Renin Angiotensin Aldosterone Syst 1: 278-282, 2000.

13. Yamada T, Kondo T, Numaguchi Y, et al: Angiotensin II receptor blocker inhibits neointimal hyperplasia through regulation of smooth muscle-like progenitor cells. Arterioscler Thromb Vasc Biol 27: 2363-2369, 2007.

14. Akishita M, Horiuchi M, Yamada H, et al: Inflammation influences vascular remodeling through AT2 receptor expression and signaling. Physiol Genomics 2: 13-20, 2000.

15. Henrion D, Kubis N and Levy BI: Physiological and pathophysiological functions of the AT(2) subtype receptor of angiotensin II: from large arteries to the microcirculation. Hypertension 38: 1150-1157, 2001

16. Hernandez Schulman I, Zhou MS and Raij L: Cross-talk between angiotensin II receptor types 1 and 2: potential role in vascular remodeling in humans. Hypertension 49: 270-271, 2007.

17. Nakajima M, Hutchinson HG, Fujinaga M, et al: The angiotensin II type 2 (AT2) receptor antagonizes the growth effects of the AT1 receptor: gain-of-function study using gene transfer. Proc Natl Acad Sci USA 92: 10663-10667, 1995.

18. Chassagne C, Adamy C, Ratajczak P, et al: Angiotensin II AT(2) receptor inhibits smooth muscle cell migration via fibronectin cell production and binding. Am J Physiol Cell Physiol 282: C654-C664, 2002.

19. Zhang C, Zhao YX, Zhang YH, et al: Angiotensin-converting enzyme 2 attenuates atherosclerotic lesions by targeting vascular cells. Proc Natl Acad Sci USA 107: 15886-15891, 2010.

20. Matsumae H, Yoshida Y, Ono K, et al: CCN1 knockdown suppresses neointimal hyperplasia in a rat artery balloon injury model. Arterioscler Thromb Vasc Biol 28: 1077-1083, 2008.

21. Cheng XW, Kuzuya M, Sasaki T, et al: Green tea catechins inhibit neointimal hyperplasia in a rat carotid arterial injury model by TIMP-2 overexpression. Cardiovasc Res 62: 594-602, 2004.

22. Yang DC, Ma ST, Tan Y, et al: Imbalance of matrix metalloproteinases/tissue inhibitor of metalloproteinase-1 and loss of fibronectin expression in patients with congestive heart failure. Cardiology 116: 133-141, 2010.

23. Yang D, Ma S, Li D, Tang B and Yang Y: Angiotensin II receptor blockade improves matrix metalloproteinases/tissue inhibitor of matrix metalloproteinase-1 balance and restores fibronectin expression in rat infarcted myocardium. Biochem Biophys Res Commun 388: 606-611, 2009.

24. Yang D, Ma S, Tan Y, et al: Increased expression of calpain and elevated activity of calcineurin in the myocardium of patients with congestive heart failure. Int J Mol Med 26: 159-164, 2010.

25. Hannan RE and Widdop RE: Vascular angiotensin II actions mediated by angiotensin II type 2 receptors. Curr Hypertens Rep 6: 117-123, 2004.

26. Levy BI: Can angiotensin II type 2 receptors have deleterious effects in cardiovascular disease? Implications for therapeutic blockade of the renin-angiotensin system. Circulation 109: 8-13, 2004.

27. Gorin Y, Ricono JM, Wagner B, et al: Angiotensin II-induced ERK1/ERK2 activation and protein synthesis are redox-dependent in glomerular mesangial cells. Biochem J 381: 231-239, 2004.

28. Stroth U, Blume A, Mielke K and Unger T: Angiotensin AT(2) receptor stimulates ERK1 and ERK2 in quiescent but inhibits ERK in NGF-stimulated PC12W cells. Brain Res Mol Brain Res 78: 175-180, 2000.

29. Kawai-Kowase K, Kurabayashi M, Hoshino Y, Ohyama Y and Nagai R: Transcriptional activation of the zinc finger transcription factor BTEB2 gene by Egr-1 through mitogen-activated protein kinase pathways in vascular smooth muscle cells. Circ Res 85: 787-795, 1999. 